\title{
Whipple's disease diagnosed during anti-tumor necrosis factor alpha treatment: two case reports and review of the literature

Jose M. Ramos ${ }^{1,2,7^{*}}$, Francisco Pasquau ${ }^{3}$, Nora Galipienso ${ }^{3}$, Beatriz Valero ${ }^{1}$, Angela Navarro ${ }^{3}$, Agustín Martinez ${ }^{4}$, José Rosas ${ }^{5}$, Ana Gutiérrez ${ }^{6}$ and Rosario Sanchez-Martínez ${ }^{1}$

\begin{abstract}
Introduction: Whipple's disease is a rare infectious disease caused by Tropheryma whipplei with protean clinical manifestations. This infection may mimic chronic inflammatory rheumatisms.

Case presentation: We report two cases of Whipple's disease diagnosed in the context of an inflammatory disease with anti-tumor necrosis factor alpha failure. The first patient was a 58-year-old white man with psoriatic spondylarthritis, who was treated with adalimumab, etanercept, infliximab, tocilizumab and golimumab. The second was a 73-year-old white man with rheumatoid arthritis, who received treatment with infliximab, then etanercept and rituximab.

Conclusions: Whipple's disease should be suspected in all patients diagnosed with chronic inflammatory rheumatism, partially controlled or not controlled by treatment with tumor necrosis factor alpha blockers, whose condition worsens after treatment.
\end{abstract}

Keywords: Whipple disease, Infliximab, Etanercept, Spondylarthritis, Rheumatoid arthritis

\section{Introduction}

Whipple's disease (WD) is a rare, chronic, systemic infection caused by Tropheryma whipplei, a Gram-positive intracellular bacillus related to actinomycetes. WD is a rare infectious disease with protean clinical manifestations. WD may often manifest itself as chronic seronegative oligoarthritis or polyarthritis, which may mimic various joint diseases (rheumatoid arthritis or spondylarthritis). The organism may be detectable by periodic acid-Schiff (PAS) staining of affected organ tissue, especially small bowel, or with $16 \mathrm{~S}$ ribosomal ribonucleic acid (rRNA) gene identification by polymerase chain reaction (PCR) amplification $[1,2]$.

Increased susceptibility to infections is a major safety concern with tumor necrosis factor alpha (TNF- $\alpha$ ) antagonist treatment [3]. Infection should be ruled out in

\footnotetext{
* Correspondence: jramosrincon@yahoo.es

'Department of Internal Medicine, Hospital General Universitario de Alicante, c/ Pintor Baeza, 12, 03010 Alicante, Spain

2Department of Medicine, Miguel Hernández University of Elche, Sant Joan d'Alacant 03550, Spain

Full list of author information is available at the end of the article
}

atypical cases by searching for foci [4]. Several authors have shown that treatment with TNF- $\alpha$ blockers seems to increase the risk of exacerbating WD [5] or worsening preexisting WD, triggering visceral disorders $[4,6]$.

We describe two cases of patients who were given TNF- $\alpha$ antagonists to treat long-standing joint disease, who then experienced the involvement of several organs leading to the diagnosis of WD. We also review the case reports of WD diagnosed during anti-TNF- $\alpha$ treatment.

\section{Case presentation}

From 2000 to 2012, eight cases of WD were diagnosed at two Spanish hospitals (Hospital General Universitario de Alicante, Alicante and Hospital Marina Baixa, Villajoyosa, Alicante), and two cases were associated with use of TNF- $\alpha$ antagonists.

\section{Case report 1}

A 58-year-old white man with inflammatory back pain and large and small joint arthritis had been diagnosed with psoriatic spondylarthritis 9 years ago. Our patient 
had been treated with adalimumab for 4 months, after that with etarnecept for 8 months, then infliximab for 2 months, tocilizumab for 21 months and golimumab for 1 month, to treat the pain in his back and neck with the consequent difficulty in bending, and arthritis of his knee and interphalangeal joint arthritis. Our patient was admitted to the hospital with abdominal septic shock. A computed tomography (CT) scan showed multiple retroperitoneal lymph nodes. The colonoscopy result was normal and the biopsy result was normal. Three months later, he was admitted again with a fever and heart failure, which was interpreted as a side effect of the golimumab treatment. One year after that, he was admitted to the hospital with abdominal pain, diarrhea and weight loss progressing to a severe wasting syndrome. At this time, he was being treated with $5 \mathrm{mg}$ prednisone plus hydroxicloroquine and methotrexate (MTX). Abnormal laboratory test results included a white blood cell (WBC) count of $14,630 / \mathrm{mm}^{3}$, a hemoglobin level of $9.6 \mathrm{~g} / \mathrm{dL}$ and an erythrocyte sedimentation rate (ESR) of $58 \mathrm{~mm} / \mathrm{h}$. A CT scan showed multiple lymph nodes. Endoscopy showed diffuse intestinal lymphangiectasia (Fig. 1). A duodenal biopsy showed distortion of the villous architecture with abundant macrophages, and bacilliform intracytoplasmic structures that stained positive with PAS with diastase digestion compatible with WD. A PCR assay for detecting T. whipplei was not done. Intravenous ceftriaxone ( $2 \mathrm{~g}$ daily for 2 weeks) was commenced followed by trimethoprim and sulphamethoxazole with improved symptoms after 3 weeks; treatment was continued for 18 months. One year later, a new gastroscopy with duodenal biopsy was done. It did not show intestinal lymphangiectasia. A PCR assay result for T. whipplei was negative. There were no relapses after 19 months.

\section{Case report 2}

A 73-year-old white man had been diagnosed with rheumatoid arthritis with migratory arthralgias of the large joints and chronic obstructive pulmonary disease

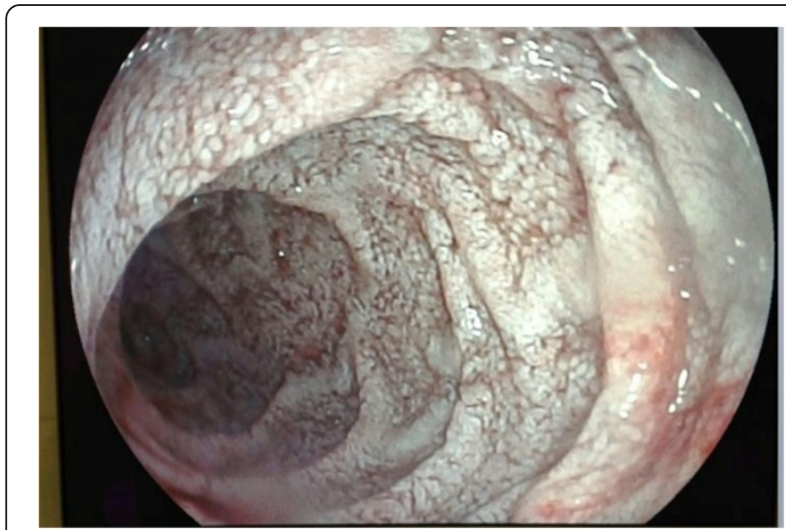

Fig. 1 Endoscopy. White lesions compatible with diffuse intestinal lymphangiectasia
14 years ago. Our patient had been treated with gold salts, chloroquine and MTX. Fourteen years after diagnosis of his diseases, infliximab was added to the MTX treatment without improvement, so infliximab was suspended 8 months later because there was no improvement of his migratory nondeforming polyarthritis; treatment with MTX was continued. After 5 months infliximab was stopped, and etanercept was added to MTX for 6 months. During treatment with etanercept, he suffered an acute middle cerebral artery ischemic stroke of atherothrombotic origin, and etanercept was stopped. Six months later, rituximab was added for 3 months, without improvement. After that, MTX was stopped and leflunomide $(20 \mathrm{mg} /$ day) was initiated and from that point, our patient presented with abdominal pain, chronic diarrhea and edema in his lower extremities, a consequence of chronic malabsorption. After 1 year on this treatment, he was admitted to hospital with rectal bleeding, however, the colonoscopy and gastroscopy results were normal and the colon biopsy showed unspecific changes. At that time, our patient was being treated with leflunomide, which was then stopped. Three months after that admission, our patient was admitted with weight loss, abdominal pain and diarrhea. On physical examination, he had hyperpigmentation of the skin but no other abnormalities. Abnormal laboratory test results included a WBC count of $13,800 / \mathrm{mm}^{3}$, a hemoglobin level of $9.2 \mathrm{~g} / \mathrm{dL}$, mean corpuscular volume (MCV) of $72 \mathrm{fl}$, an albumin level of $1.8 \mathrm{~g} / \mathrm{dL}$, and an ESR of $13 \mathrm{~mm} / \mathrm{h}$. A thoracic and abdominal CT scan showed pericardial effusion with calcifications, bronchiectasis in his lower right lung, intestinal bowel with distention and no abdominal lymph nodes. A duodenal biopsy showed altered architecture and intracellular bacilli on PAS stain. T. whipplei was detected from duodenal tissue by PCR assay. A cerebral magnetic resonance imaging scan showed multiple hyperintensive lesions in both cerebral hemispheres, cortical retraction, increased subarachnoid space and ventricular dilatation. The PCR assay result for T. whipplei in his cerebrospinal fluid was negative. Intravenous ceftriaxone ( $2 \mathrm{~g}$ daily) was commenced for 2 weeks followed by trimethoprim and sulphamethoxazole with improvement of his symptoms (the diarrhea, malabsorption and pericardial effusion). One year later, a new gastroscopy with duodenal biopsy was done. It showed altered architecture and intracellular bacilli on PAS stain, but the PCR assay result for $T$. whipplei was negative. Because of mild renal failure, trimethoprim and sulphamethoxazole was changed for doxycycline plus hydroxychloroquine, and normal renal function was recovered.

\section{Discussion}

We reviewed database cases recorded in PubMed using the following retrieval scheme: ["Whipple disease" and 
("infliximab" or "adalimumab" or "etanercept" or "golimumab" or "tocilizumab")]. We gathered the following data from the medical cases reported: age, sex, joint diseases, years with joint disease, TNF- $\alpha$ antagonist therapy, days with TNF- $\alpha$ antagonist therapy before WD was diagnosed, symptoms related to WD, organs affected by WD, investigations for diagnosing WD, treatment and outcome of WD.

We retrieved 14 cases from the PubMed database from January 2004 to December 2014. All the case reports recorded and the two case reports in this manuscript are from European scientists [4-10], except one case from the United States of America [11]. Four case reports were published in a language other than English $[7,8,10]$. Table 1 shows age, sex, joint diseases, years with joint disease, TNF- $\alpha$ antagonist therapy, days with TNF- $\alpha$ antagonist therapy before WD was diagnosed, symptoms related to WD, organs affected by WD, investigations for diagnosing WD, treatment and outcome of WD for 16 WD cases. Out of 16 cases, there were 14 men and two women patients with an age range of 33 to 73 years old. Six patients were diagnosed as ankylosis spondylitis (AS), six as seronegative spondyloarthropathy (SA) (seronegative chronic polyarthritis), two as rheumatoid arthritis (RA), one as Still's disease (SD), and one as psoriatic arthritis. All patients had osteoarticular (with pain and swelling) involvement and 15 had gastrointestinal involvement (diarrhea, weight loss, abdominal pain, malabsorption, and so on) [4-12]. Moreover, some patients had extra-articular and gastrointestinal involvement, such as vertebra [5], meningitis [6], pericarditis ([6], present report 2 (PR2)), abdominal or thoracic lymph nodes $([4,6], \mathrm{PR} 1)$, gingiva such as scurvy [9] and heart valve involvement [10]. All were diagnosed by histological biopsy, in 14 of 15 cases a PCR assay for DNA detection of T. whipplei was done, and all were positive. The DNA of T. whipplei by PCR assay was detected in duodenal or other gastrointestinal-colonic areas; saliva, blood, feces, bone or cerebrospinal fluid [4-11]. All patients recovered from gastrointestinal involvement when the TNF- $\alpha$ antagonist was stopped and antibiotic treatment was started.

\section{Conclusions}

WD is a systemic infection, which involves many chronic manifestations, especially digestive disorders. Some of the manifestations involved are articular symptoms, which can appear as inflammatory rheumatism, such as rheumatoid arthritis or spondylarthritis. Beside these symptoms, the microorganism responsible for $T$. whipplei disease has also been found in DNA recovered from articular fluid and bones of these patients $[13,14]$.

Our two cases illustrate that alternative etiologies in patients with articular symptoms should be considered, most importantly following clinical deterioration by immunomodulatory agents like TNF- $\alpha$ inhibitors. The reflections to be made with respect to the analysis of these two cases are to speculate that these patients either had WD with mainly articular manifestations, which had been considered as a rheumatic disease; or in fact, they had a rheumatic disease, which, through the treatment with TNF drugs, may have reactivated T. whipplei and the appearance, therefore, of its florid symptoms that we managed to diagnose.

Therapies with immunomodulators, TNF- $\alpha$ inhibitors, and corticosteroids may transform an infection with $T$. whipplei, normally at a subacute stage, into a septic, life-threatening disease. Thus, it seems that TNF- $\alpha$ blockade allowed for rapid dissemination of T. whipplei by inhibiting some important immune defense mechanisms. In the later phase of an infection, the TNF- $\alpha$ blocker contributes to limiting the extent of cell and/or tissue damage by inducing apoptosis and maintaining granuloma formation $[15,16]$, which are important mechanisms in T. whipplei infections. TNF- $\alpha$ blockade might result in the loss of some of the immunological control mechanisms and, therefore, facilitate rapid bacterial dissemination and severe exacerbation of the disease. This is the situation in the first case.

In the second case, the TNF- $\alpha$ blockade was used before the gastrointestinal symptoms appeared. However, with anti-TNF- $\alpha$ treatment, our patient had an acute stroke. After stopping the treatment, our patient recovered from his central nervous system symptoms. When the gastrointestinal involvement of WD was discovered, an examination of DNA for T. whipplei was performed but it was negative. In this case, it was not clear that our patient had neurological involvement of WD, and real improvement of WD after stopping the TNF- $\alpha$ blockade treatment was not clear. There are several cases reported of patients with rheumatic diseases and, during follow-up, they were diagnosed with WD [13, 14, 17, 18]. Thus, the case report of a patient with an initial diagnosis of rheumatoid arthritis who developed pericarditis caused by WD (diagnosed by pericardial biopsy) and the patient who had not been treated previously with TNF- $\alpha$ blocker [17].

In summary, anti-TNF- $\alpha$ treatment seems to increase the risk of exacerbation of WD and WD may mimic a rheumatic disease.

\section{Consent}

Written informed consent was obtained from the patients for publication of this case report and any accompanying images. A copy of the written consent is available for review by the Editor-in-Chief of this journal. 
Table 1 Features of the patients' diagnosis of Whipple's disease after tumor necrosis factor alpha antagonist initiation

\begin{tabular}{|c|c|c|c|c|c|c|c|c|c|c|}
\hline $\begin{array}{l}\text { Authors / country / } \\
\text { language of } \\
\text { publication }\end{array}$ & Age / sex & $\begin{array}{l}\text { Underlying disease } \\
\text { previous WD } \\
\text { diagnosis / time } \\
\text { with that diagnosis }\end{array}$ & $\begin{array}{l}\text { TNF-a drugs and } \\
\text { immunosuppressive } \\
\text { treatment }\end{array}$ & $\begin{array}{l}\text { Start of acute } \\
\text { symptoms after } \\
\text { TNF-a blockade }\end{array}$ & $\begin{array}{l}\text { Symptoms after TNF-a } \\
\text { blockade }\end{array}$ & Organ involvement & $\begin{array}{l}\text { Histological } \\
\text { diagnosis }^{a}\end{array}$ & $\begin{array}{l}\text { Result of PCR } \\
\text { for Tropheryma } \\
\text { whipplei }\end{array}$ & $\begin{array}{l}\text { Treatment } \\
\text { for WD }\end{array}$ & Outcome \\
\hline $\begin{array}{l}\text { Kneitz et al. } \\
(2005) \text { [4] / } \\
\text { Germany / } \\
\text { English }\end{array}$ & $34 \mathrm{y} / \mathrm{M}$ & SD & $\begin{array}{l}\text { Methotrexate + } \\
\text { infliximab }(2 \mathrm{w})\end{array}$ & $2 w$ & $\begin{array}{l}\text { Weight loss, erythema } \\
\text { nodosum, diarrhea, } \\
\text { lymph node } \\
\text { enlargement, and } \\
\text { a sigmoidovesical } \\
\text { fistula }\end{array}$ & $\begin{array}{l}\text { Skin, gastroduodenitis } \\
\text { and sigmoidovesical }\end{array}$ & $\begin{array}{l}\text { Positive in } \\
\text { fistula and } \\
\text { lymph nodes }\end{array}$ & $\begin{array}{l}\text { Positive in } \\
\text { lymph node, } \\
\text { small bowel } \\
\text { and } \\
\text { sigmoidovesical } \\
\text { fistula }\end{array}$ & $\mathrm{SXT}(12 \mathrm{~m})$ & $\begin{array}{l}\text { Resolved } \\
\text { within } \\
1 \text { year }\end{array}$ \\
\hline $\begin{array}{l}\text { Kremer et al. } \\
\text { (2008) [7] / } \\
\text { Germany / } \\
\text { German }\end{array}$ & $47 y / M$ & SA / 4y & $\begin{array}{l}\text { Leflunomide + } \\
\text { adalimumab }\end{array}$ & & $\begin{array}{l}\text { Fever, weight loss, } \\
\text { and severe arthralgia }\end{array}$ & & $\begin{array}{l}\text { Positive in } \\
\text { duodenal } \\
\text { tissue }\end{array}$ & $\begin{array}{l}\text { Positive in } \\
\text { duodenal tissue }\end{array}$ & SXT & \\
\hline $\begin{array}{l}\text { Spoerl et al. } \\
\text { (2009) [5] / } \\
\text { Switzerland / } \\
\text { English }\end{array}$ & $64 \mathrm{y} / \mathrm{M}$ & $S A / 5+3 y$ & Etanercept & $4 m$ & $\begin{array}{l}\text { Lethargy, night } \\
\text { sweats, weight loss }\end{array}$ & $\begin{array}{l}\text { Gastrointestinal } \\
\text { and vertebral }\end{array}$ & $\begin{array}{l}\text { Positive in } \\
\text { duodenal } \\
\text { tissue and } \\
\text { vertebral } \\
\text { tissue }\end{array}$ & $\begin{array}{l}\text { Positive in } \\
\text { gastric and } \\
\text { vertebral tissue }\end{array}$ & $\begin{array}{l}\text { Ceftriaxone } \\
(2 \mathrm{w}), \text { then, } \\
\text { SXT }(2 \mathrm{y}) \text {, } \\
(12 \mathrm{~m}) \text { then } \\
\text { doxycycline + } \\
\text { HC }(18 \mathrm{~m})\end{array}$ & $\begin{array}{l}\text { No relapses } \\
\text { after } \\
34-\text { month } \\
\text { follow-up }\end{array}$ \\
\hline $\begin{array}{l}\text { Ahmadi-Simab } \\
\text { et al. (2009) } \\
\text { [8] / Germany / } \\
\text { German }\end{array}$ & $33 y / F$ & AS / 8y & Etanercept & $12 m$ & $\begin{array}{l}\text { Diarrhea, abdominal } \\
\text { pain and weight loss }\end{array}$ & & $\begin{array}{l}\text { Negative in } \\
\text { duodenal } \\
\text { tissue }\end{array}$ & $\begin{array}{l}\text { Positive in } \\
\text { duodenal } \\
\text { biopsy }\end{array}$ & $\begin{array}{l}\text { Ceftriaxone } \\
(2 w) \text { after SXT }\end{array}$ & \\
\hline $\begin{array}{l}\text { Hoppé et al. } \\
\text { (2010) [6] / } \\
\text { France / English }\end{array}$ & $67 y / F$ & SA / 7y & Infliximab (4m) & $4 m$ & $\begin{array}{l}\text { Chest pain, dyspnea, } \\
\text { polyarthritis }\end{array}$ & & $\begin{array}{l}\text { Positive in } \\
\text { duodenal } \\
\text { tissue }\end{array}$ & $\begin{array}{l}\text { Positive in } \\
\text { duodenal } \\
\text { tissue. Negative } \\
\text { in blood and } \\
\text { saliva }\end{array}$ & $\begin{array}{l}\text { Ceftriaxone } \\
(2 \mathrm{w}) \text {, then } \\
\text { doxycycline } \\
(24 \mathrm{~m})\end{array}$ & $\begin{array}{l}\text { Resolved } \\
\text { within } 15 \mathrm{~d} \text {. } \\
\text { No relapses } \\
\text { after } \\
71 \text {-month } \\
\text { follow-up }\end{array}$ \\
\hline $\begin{array}{l}\text { Hoppé et al. } \\
\text { (2010) [6] / } \\
\text { France / English }\end{array}$ & $40 y / M$ & AS / 1y & $\begin{array}{l}\text { Infliximab }(18 \mathrm{~m}) \\
\text { then etanercept } \\
(7 \mathrm{~m}) \text { then } \\
\text { adalimumab }(1 \mathrm{~m})\end{array}$ & $26 \mathrm{~m}$ & $\begin{array}{l}\text { Diarrhea, weight loss, } \\
\text { fever, arthralgia }\end{array}$ & $\begin{array}{l}\text { Widespread } \\
\text { ileocolitis, } \\
\text { gastroduodenitis, } \\
\text { meningitis }\end{array}$ & $\begin{array}{l}\text { Positive in } \\
\text { duodenal } \\
\text { tissue }\end{array}$ & $\begin{array}{l}\text { Positive in } \\
\text { saliva, feces } \\
\text { and } \\
\text { cerebrospinal } \\
\text { fluid. Negative } \\
\text { in blood }\end{array}$ & $\begin{array}{l}\text { Doxycycline, } \\
\text { HC, and SXT } \\
(15 d) \text {, then } \\
\text { SXT was } \\
\text { replaced by } \\
\text { sulfasalazine } \\
4 \mathrm{~g} / \text { day }\end{array}$ & $\begin{array}{l}\text { Resolved } \\
\text { within } \\
2 \text { months. } \\
\text { No relapses } \\
\text { after } \\
17 \text {-month } \\
\text { follow-up }\end{array}$ \\
\hline $\begin{array}{l}\text { Hoppé et al. } \\
\text { (2010) [6] / } \\
\text { France / English }\end{array}$ & $60 y / M$ & AS / 8y & Etanercept $(9 \mathrm{~m})$ & $9 m$ & $\begin{array}{l}\text { Fever, night sweats, } \\
\text { polyarthritis, } \\
\text { chest pain }\end{array}$ & $\begin{array}{l}\text { Pericarditis, } \\
\text { mediastinal and } \\
\text { abdominal } \\
\text { lymphadenopathy, } \\
\text { duodenitis }\end{array}$ & $\begin{array}{l}\text { Negative in } \\
\text { duodenal } \\
\text { tissue }\end{array}$ & $\begin{array}{l}\text { Positive in } \\
\text { the duodenal } \\
\text { sample, saliva, } \\
\text { feces, and } \\
\text { lymph nodes }\end{array}$ & $\begin{array}{l}\text { Doxycycline + } \\
\text { HC }(15 \mathrm{~m})\end{array}$ & $\begin{array}{l}\text { Resolved } \\
\text { within } 7 \mathrm{~d} \text {. } \\
\text { No relapses } \\
\text { after } \\
\text { 30-month } \\
\text { follow-up }\end{array}$ \\
\hline
\end{tabular}


Table 1 Features of the patients' diagnosis of Whipple's disease after tumor necrosis factor alpha antagonist initiation (Continued)

\begin{tabular}{|c|c|c|c|c|c|c|c|c|c|c|}
\hline $\begin{array}{l}\text { Hoppé et al. } \\
\text { (2010) [6] / } \\
\text { France / } \\
\text { English }\end{array}$ & $47 y / M$ & RA / 17y & $\begin{array}{l}\text { Infliximab }(36 \mathrm{~m}) \text {, } \\
\text { then etanercept } \\
(42 \mathrm{~m}), \text { then } \\
\text { adalimumab }(6 \mathrm{~m}) \text {, } \\
\text { then rituximab, } \\
\text { abatacept then } \\
\text { infliximab }(1 \mathrm{~m})\end{array}$ & $85 m$ & $\begin{array}{l}\text { Fever, night sweats, } \\
\text { weight loss, } \\
\text { polyarthritis, radio } \\
\text { carpal arthropathy. } \\
\text { transient diplopia, } \\
\text { myalgia, subacute } \\
\text { depression }\end{array}$ & $\begin{array}{l}\text { Pericarditis, abdominal } \\
\text { lymphadenopathy, } \\
\text { duodenitis }\end{array}$ & & $\begin{array}{l}\text { Positive PCR } \\
\text { assay in the } \\
\text { duodenal } \\
\text { sample, blood, } \\
\text { saliva, and } \\
\text { feces. Negative } \\
\text { PCR assay in } \\
\text { cerebrospinal } \\
\text { fluid }\end{array}$ & $\begin{array}{l}\text { Doxycycline + } \\
\text { HC (UT) }\end{array}$ & $\begin{array}{l}\text { Resolved } \\
\text { within } 2 \mathrm{~m} \\
\text { No relapses } \\
\text { after } \\
\text { 15-month } \\
\text { follow-up }\end{array}$ \\
\hline $\begin{array}{l}\text { Hoppé et al. } \\
\text { (2010) [6] / } \\
\text { France / } \\
\text { English }\end{array}$ & $38 y / M$ & AS / 9y & Etanercept (2m) & $9 m$ & $\begin{array}{l}\text { Diarrhea, abdominal } \\
\text { pain. weight loss, } \\
\text { blurred vision }\end{array}$ & $\begin{array}{l}\text { Hemorrhagic } \\
\text { gastroduodenitis, } \\
\text { hemorrhagic } \\
\text { colitis, mediastinal } \\
\text { and cervical } \\
\text { lymphadenopathy, } \\
\text { splenomegaly, } \\
\text { meningitis }\end{array}$ & $\begin{array}{l}\text { Positive in } \\
\text { duodenum } \\
\text { and colon }\end{array}$ & $\begin{array}{l}\text { Positive PCR } \\
\text { assay in blood, } \\
\text { saliva, } \\
\text { cerebrospinal } \\
\text { fluid, and feces }\end{array}$ & $\begin{array}{l}\text { Doxycycline } \\
\text { 200mg/day. } \\
\text { HC 600mg/ } \\
\text { day. (UT) }\end{array}$ & $\begin{array}{l}\text { Resolved } \\
\text { within } 3 \mathrm{w} \text {. } \\
\text { No relapses } \\
\text { after } \\
\text { 13-month } \\
\text { follow-up }\end{array}$ \\
\hline $\begin{array}{l}\text { Hmamouchi } \\
\text { et al. (2010) [9] / } \\
\text { France / English }\end{array}$ & $35 y / M$ & AS / $4 y$ & Etanercept & $4 m$ & $\begin{array}{l}\text { Gingival nodule, } \\
\text { purpura, abdominal } \\
\text { pain diarrhea, fatigue, } \\
\text { weight loss }\end{array}$ & Gingiva and colitis & $\begin{array}{l}\text { Positive in } \\
\text { colon tissue }\end{array}$ & $\begin{array}{l}\text { Positive in } \\
\text { blood, stool, } \\
\text { and } \\
\text { cerebrospinal } \\
\text { fluid }\end{array}$ & SXT (NR) & $\begin{array}{l}\text { Resolved } \\
\text { within } \\
3 \text { months }\end{array}$ \\
\hline $\begin{array}{l}\text { Daïen et al. } \\
\text { (2010) [10] / } \\
\text { France / English }\end{array}$ & $70 y / M$ & SA / 27y & Etanercept (18m) & NR & Fever and dyspnea & $\begin{array}{l}\text { Endocarditis of } \\
\text { aortic valve }\end{array}$ & $\begin{array}{l}\text { Negative in } \\
\text { duodenal } \\
\text { tissue and, } \\
\text { aortic valve }\end{array}$ & $\begin{array}{l}\text { Positive in } \\
\text { aortic valve }\end{array}$ & $\begin{array}{l}\text { Doxycycline } \\
\mathrm{HC}+\mathrm{SXT} \\
(18 \mathrm{~m})\end{array}$ & $\begin{array}{l}\text { Resolved. } \\
\text { No relapses } \\
\text { after } \\
21 \text {-month } \\
\text { follow-up }\end{array}$ \\
\hline $\begin{array}{l}\text { Gaddy et al., } \\
\text { (2012) [12]/ } \\
\text { USA / English }\end{array}$ & $46 y / M$ & AS / 10y & $\begin{array}{l}\text { Prednisone + } \\
\text { MTX + infliximab } \\
(24 \mathrm{~m}), \text { then } \\
\text { adalimumab }\end{array}$ & $24 m$ & $\begin{array}{l}\text { Fever, migratory } \\
\text { arthritis, weight loss, } \\
\text { diarrhea }\end{array}$ & Gastroenteritis & $\begin{array}{l}\text { Positive in } \\
\text { duodenal } \\
\text { tissue }\end{array}$ & $\begin{array}{l}\text { Positive in } \\
\text { blood and } \\
\text { duodenal } \\
\text { tissue }\end{array}$ & $\begin{array}{l}\text { Ceftriaxone } \\
(\times 2 w) \text { after } \\
\text { SXT }\end{array}$ & NR \\
\hline $\begin{array}{l}\text { Sparsa et al. } \\
\text { (2013) [11] / } \\
\text { France / French }\end{array}$ & $53 y / M$ & SA & $\begin{array}{l}\text { Etanercept }(3 \mathrm{~m}), \\
\text { then adalimumab } \\
(6 \mathrm{~m})\end{array}$ & $9 m$ & $\begin{array}{l}\text { Polyarthralgia } \\
\text { peripheric, arthritis } \\
\text { metarsophalangeal }\end{array}$ & & $\begin{array}{l}\text { Positive in } \\
\text { duodenal } \\
\text { tissue biopsy }\end{array}$ & $\begin{array}{l}\text { Positive in } \\
\text { saliva and } \\
\text { duodenal } \\
\text { tissue }\end{array}$ & $\begin{array}{l}\text { Doxycycline + } \\
\text { HC }(18 \mathrm{~m})\end{array}$ & $\begin{array}{l}\text { Resolved } \\
\text { within } \\
3 \text { weeks. No } \\
\text { relapses } \\
\text { after } \\
\text { 18-month } \\
\text { follow-up }\end{array}$ \\
\hline $\begin{array}{l}\text { Sparsa et al. } \\
\text { (2013) [11] / } \\
\text { France / French }\end{array}$ & $42 y / M$ & $\mathrm{SA} / 2 \mathrm{~m}$ & $\begin{array}{l}\text { Adalimumab } \\
(9 \mathrm{~m}) \text { and then } \\
\text { etanercept }(4 \mathrm{~m})\end{array}$ & $13 m$ & $\begin{array}{l}\text { Polyarthralgia } \\
\text { peripheric }\end{array}$ & & $\begin{array}{l}\text { Positive in } \\
\text { duodenal } \\
\text { and gastric } \\
\text { tissue }\end{array}$ & $\begin{array}{l}\text { Positive in } \\
\text { saliva and } \\
\text { duodenal } \\
\text { tissue }\end{array}$ & $\begin{array}{l}\text { Doxycycline + } \\
\mathrm{HC} \text { and then } \\
\text { doxycycline } \\
\text { alone }\end{array}$ & $\begin{array}{l}\text { Resolved } \\
\text { within } \\
3 \text { weeks. } \\
\text { No relapses } \\
\text { after } \\
17-\text { month } \\
\text { follow-up }\end{array}$ \\
\hline
\end{tabular}


Table 1 Features of the patients' diagnosis of Whipple's disease after tumor necrosis factor alpha antagonist initiation (Continued)

\begin{tabular}{|c|c|c|c|c|c|c|c|c|c|c|}
\hline Present report & $58 y / M$ & $\begin{array}{l}\text { Psoriatic } \\
\text { spondyloarthropathy/ } \\
14 y\end{array}$ & $\begin{array}{l}\text { Adalimumab }(4 \mathrm{~m}) \text {, } \\
\text { then etanercept } \\
(8 \mathrm{~m}) \text {, infliximab } \\
(2 \mathrm{~m}) \text {, tocilizumab } \\
(21 \mathrm{~m}) \text {, golimumab } \\
(1 \mathrm{~m})\end{array}$ & $36 \mathrm{~m}$ & $\begin{array}{l}\text { Abdominal pain, } \\
\text { diarrhea and weight } \\
\text { loss }\end{array}$ & $\begin{array}{l}\text { Duodenitis and } \\
\text { abdominal lymph } \\
\text { nodes }\end{array}$ & $\begin{array}{l}\text { Positive in } \\
\text { duodenal } \\
\text { tissue }\end{array}$ & Not done & $\begin{array}{l}\text { Ceftriaxone } \\
\text { (14d), then } \\
\text { SXT during } \\
\text { (UT) }\end{array}$ & $\begin{array}{l}\text { Resolved } \\
\text { within } \\
3 \text { weeks. } \\
\text { No relapses } \\
\text { after 9-month } \\
\text { follow-up. }\end{array}$ \\
\hline Present report & $78 y / M$ & RA / 19y & $\begin{array}{l}\text { Infliximab }(6 \mathrm{~m}), \\
\text { etanercept }(6 \mathrm{~m}), \\
\text { rituximab }(3 \mathrm{~m}), \\
\text { leflunomide }(24 \mathrm{~m})\end{array}$ & $24 m$ & $\begin{array}{l}\text { Abdominal pain, } \\
\text { diarrhea and weight } \\
\text { loss }\end{array}$ & $\begin{array}{l}\text { Duodenitis, and } \\
\text { pericardial effusion }\end{array}$ & $\begin{array}{l}\text { Positive in } \\
\text { duodenal } \\
\text { tissue }\end{array}$ & $\begin{array}{l}\text { Positive in } \\
\text { duodenal } \\
\text { biopsy. } \\
\text { Negative in } \\
\text { cerebrospinal } \\
\text { fluid }\end{array}$ & $\begin{array}{l}\text { Ceftriaxone } \\
(14 d) \text {, then } \\
\text { SXT for } \\
(12 \mathrm{~m}) \text { then } \\
\text { doxycycline + } \\
\text { HC }\end{array}$ & $\begin{array}{l}\text { Resolved } \\
\text { within } \\
3 \text { weeks. } \\
\text { No relapses } \\
\text { after } \\
18 \text {-month } \\
\text { follow-up }\end{array}$ \\
\hline
\end{tabular}

(UT) Patient still under treatment

TNF- $a$ tumor necrosis factor alpha, WD Whipple's disease, PCR polymerase chain reaction, y year, SD Still's disease, $w$ week, SXT cotrimoxazole, $m$ month, SA spondyloarthropathy (seronegative chronic polyarthritis) $H C$ hydroxychlorochine, $A S$ ankylosis spondylitis, RA rheumatoid arthritis, NR not reported, MTX methotrexate

aistological diagnosis $=$ intracellular bacilli on periodic acid-Schiff stain 


\section{Abbreviations}

AS: Ankylosis spondylitis; CT: Computed tomography; ESR: Erythrocyte sedimentation rate; HC: Hydroxychlorochine; m: month; MCV: Mean corpuscular volume; MTX: Methotrexate; NR: Not reported; PAS: Periodic acid-Schiff; PR: Present report; PCR: Polymerase chain reaction; RA: Rheumatoid arthritis; SA: Spondyloarthropathy (seronegative chronic polyarthritis); SD: Still's diseases; SXT: Cotrimoxazole; rRNA: ribosomal ribonucleic acid; TNF-a: Tumor necrosis factor alpha; w: week; WBC: White blood cell; WD: Whipple's disease; y: year.

\section{Competing interests}

The authors declare that they have no competing interests.

\section{Authors' contributions}

All authors treated the patients during the years 2002 to 2014. Each of them has made substantial contributions to data acquisition and interpretation. All of them have been involved in drafting the manuscript. All authors read and approved the final manuscript.

\section{Acknowledgements}

We should like to acknowledge the personnel from the Department of Internal Medicine Rheumatology and Gastroenterology of the Hospital General Universitario de Alicante and the Hospital Marina Baixa, Villajoyosa, Alicante (Spain) for attending to the patients.

\section{Author details}

'Department of Internal Medicine, Hospital General Universitario de Alicante, c/ Pintor Baeza, 12, 03010 Alicante, Spain. ${ }^{2}$ Department of Medicine, Miguel Hernández University of Elche, Sant Joan d'Alacant 03550, Spain.

${ }^{3}$ Department of Internal Medicine, Hospital Marina Baixa, Avenida Alcalde Jaume Botella Mayor, 7, Villajoyosa 03570, Alicante, Spain. ${ }^{4}$ Department of Rheumatology, Hospital General Universitario de Alicante, c/ Pintor Baeza, 12, 03010 Alicante, Spain. ${ }^{5}$ Department of Rheumatology, Hospital Marina Baixa, Avenida Alcalde Jaume Botella Mayor, 7, Villajoyosa 03570, Alicante, Spain. ${ }^{6}$ Department of Gastroenterology, Hospital General Universitario de Alicante, c/ Pintor Baeza, 12, 03010 Alicante, Spain. ' Servicio de Medicina Interna, Hospital General Universitario de Alicante, c/ Pintor Baeza, 12, 03010 Alicante, Spain.

Received: 7 January 2015 Accepted: 3 June 2015

Published online: 28 July 2015

\section{References}

1. Fenollar F, Puéchal X, Raoult D. Whipple's disease. N Engl J Med. 2007;356:55-66.

2. Schneider T, Moos V, Loddenkemper C, Marth T, Fenollar F, Raoult D. Whipple's disease: new aspects of pathogenesis and treatment. Lancet Infect Dis. 2008;8:179-90.

3. Rosenblum H, Amital H. Anti-TNF therapy: safety aspects of taking the risk. Autoimmun Rev. 2011;10:563-8

4. Kneitz C, Suerbaum S, Beer M, Müller J, Jahns R, Tony HP. Exacerbation of Whipple's disease associated with infliximab treatment. Scand J Rheumatol. 2005;34:148-51.

5. Spoerl D, Bär D, Cooper J, Vogt T, Tyndall A, Walker UA. Multisegmental spondylitis due to Tropheryma whipplei: case report. Orphanet J Rare Dis. 2009:4:13.

6. Hoppé E, Masson C, Audran M, Drillon M, Andreu M, Saraux A, et al. Whipple's disease diagnosed during biological treatment for joint disease. Joint Bone Spine. 2010;77:335-9.

7. Kremer AE, Budenhofer U, Beuers U, Rust C. A 47-year-old dog breeder with chronic polyarthritis, weight loss and high fever. Z Gastroenterol. 2008; $46: 431-4$

8. Ahmadi-Simab K, Schnitzler P, Schnitzler P. Whipple's disease with normal duodenal histology and ankylosing spondylitis. Dtsch Med Wochenschr. 2009:134:127-30.

9. Hmamouchi I, Costes V, Combe B, Morel J. Scurvy as the presenting illness of Whipple's disease exacerbated by treatment with etanercept in a patient with ankylosing spondylitis. J Rheumatol. 2010;37:1077-8.

10. Daïen $\mathrm{Cl}$, Cohen JD, Makinson A, Battistella P, Bilak EJ, Jorgensen C, et al. Whipple's endocarditis as a complication of tumour necrosis factor-alpha antagonist treatment in a man with ankylosing spondylitis. Rheumatology (Oxford). 2010;49:1600-2.

11. Sparsa L, Fenollar F, Gossec L, Leone J, Pennaforte JL, Dougados M, et al. Whipple disease revealed by anti-TNFa therapy. Rev Med Interne. 2013;34:105-9.

12. Gaddy JR, Khan ZZ, Chaser B, Scofield RH. Whipple's disease diagnosis following the use of TNF-a blockade. Rheumatology (Oxford). 2012;51:946.

13. O'Duffy JD, Griffing WL, Li CY, Abdelmalek MF, Persing DH. Whipple's arthritis: direct detection of Tropheryma whipplei in synovial fluid and tissue. Arthritis Rheum. 1999;42:812-7.

14. Weber U, Morf MH, Gubler JG, Altwegg M, Maibach RC. Spondylodiscitis as the first manifestation of Whipple's disease: a removal worker with chronic low back pain. Clin Rheumatol. 2003;22:443-6.

15. Keane J, Gershon S, Wise RP, Mirabile-Levens E, Kasznica J, Schwieterman $W D$, et al. Tuberculosis associated with infliximab, a tumor necrosis factor alpha-neutralizing agent. New Engl J Med. 2001;345:1098-104.

16. Cunnane G, Doran M, Bresnihan B. Infections and biological therapy in rheumatoid arthritis. Best Pract Res Clin Rheumatol. 2003;17:345-63.

17. Makol A, Maleszewski JJ, Warrington KJ. A case of refractory rheumatoid pericarditis. Arthritis Care Res (Hoboken). 2012;64:935-40.

18. von Gerstenbergk C, Lorenz HM, Blank N. Fever and arthritis: rheumatic or Whipple's disease? Dtsch Med Wochenschr. 2011;136:1656-9.

\section{Submit your next manuscript to BioMed Central and take full advantage of:}

- Convenient online submission

- Thorough peer review

- No space constraints or color figure charges

- Immediate publication on acceptance

- Inclusion in PubMed, CAS, Scopus and Google Scholar

- Research which is freely available for redistribution

Submit your manuscript at www.biomedcentral.com/submit 